\title{
Benchmark calculations for elastic fermion-dimer scattering
}

\author{
Shahin Bour ${ }^{a}$, H.-W. Hammer ${ }^{a}$, Dean Lee ${ }^{b}$, Ulf-G. Meißner ${ }^{a, c}$ \\ ${ }^{a}$ Helmholtz-Institut für Strahlen- und Kernphysik and Bethe Center for Theoretical Physics, \\ Universität Bonn, D-53115 Bonn, Germany \\ ${ }^{b}$ Department of Physics, North Carolina State University, Raleigh, NC 27695, USA \\ ${ }^{c}$ Institut für Kernphysik, Institute for Advanced Simulation, \\ JARA-HPC and Jülich Center for Hadron Physics, \\ Forschungszentrum Jülich, D-52425 Jülich, Germany
}

\begin{abstract}
We present continuum and lattice calculations for elastic scattering between a fermion and a bound dimer in the shallow binding limit. For the continuum calculation we use the SkorniakovTer-Martirosian (STM) integral equation to determine the scattering length and effective range parameter to high precision. For the lattice calculation we use the finite-volume method of Lüscher. We take into account topological finite-volume corrections to the dimer binding energy which depend on the momentum of the dimer. After subtracting these effects, we find from the lattice calculation $\kappa a_{f d}=1.174(9)$ and $\kappa r_{f d}=-0.029(13)$. These results agree well with the continuum values $\kappa a_{f d}=1.17907(1)$ and $\kappa r_{f d}=-0.0383(3)$ obtained from the STM equation. We discuss applications to cold atomic Fermi gases, deuteron-neutron scattering in the spin-quartet channel, and lattice calculations of scattering for nuclei and hadronic molecules at finite volume.
\end{abstract}

PACS numbers: 21.60.De, 25.40.Dn, 12.38.Gc, 03.65.Ge 


\section{INTRODUCTION}

In this paper, we perform the first benchmark of finite-volume lattice methods for the low-energy scattering of composite objects. Our results will have immediate applications to lattice studies of elastic neutron-nucleus scattering. In the analysis presented here we consider scattering between a fermion and a bound dimer composed of two fermions. In order to test the precision of our lattice calculations, we also repeat the same calculations using the Skorniakov-Ter-Martirosian (STM) integral equation [1]. Along the way we also provide the most accurate calculation to date for the fermion-dimer effective range parameter. Some of the results presented were summarized in a letter publication [2], and we present the full details of the calculations here.

We consider two component fermions. We will refer to the two fermion components as spin up and spin down and consider the case when the masses are equal, $m_{\uparrow}=m_{\downarrow}$. We assume finite-range attractive interactions and consider the universal shallow binding limit. If $R$ is the range of the interactions and $\kappa$ is the binding momentum of the dimer, then the shallow binding limit corresponds to $\kappa R \rightarrow 0$.

We note that much of the literature on universal three-body systems has focused on the Efimov effect [3] for three bosons, three-component fermions, unequal mass fermions, or mixed Bose-Fermi systems [4-8]. For equal mass two-component fermions, however, there are no short-distance three-body instabilities such as the Thomas collapse [9]. Hence there are no relevant momentum scales other than the dimer binding momentum $\kappa$, and all low-energy scattering parameters can be expressed as dimensionless constants times the corresponding power of $\kappa$. In the shallow binding limit $\kappa$ is the same as the reciprocal of the fermion-fermion scattering length.

There have been numerous calculations of the fermion-dimer scattering length. The first goes back to the early work of Skorniakov and Ter-Martirosian who found $\kappa a_{f d} \approx 1.2[1]$. An overview of the Skorniakov-Ter-Martirosian (STM) integral equation method will be presented in our discussion below. The same value $\kappa a_{f d} \approx 1.2$ has been confirmed several times using integral equations [10, 11]. A value of $\kappa a_{f d} \approx 1.11$ was obtained using an epsilon expansion in dimensions [12]. A recent correlated Gaussian expansion calculation obtained $\kappa a_{f d} \approx 1.18(1)[13]$. This agrees with integral equation studies which found $\kappa a_{f d} \approx 1.18$ [14, 15] and $\kappa a_{f d} \approx 1.1790662349$ [16].

The fermion-dimer results at shallow binding should approximately describe neutrondeuteron scattering in the spin-quartet channel. Experimental measurements find a quartet scattering length ${ }^{4} a_{n d}=6.35(2) \mathrm{fm}$ [17]. This corresponds with $\kappa^{4} a_{n d}=1.47(1)$. The agreement is better when expressed as fraction of the spin-triplet proton-neutron scattering length, ${ }^{4} a_{n d} /{ }^{3} a_{n p}=1.17(1)$. The $30 \%$ difference between the two values gives an indication of higher order effective range effects. A more detailed calculation including interaction range effects obtains ${ }^{4} a_{n d}=6.33(10) \mathrm{fm}[18,19]$, in full agreement with experimental values.

In contrast with the scattering length, there is only one previously reported determination of the fermion-dimer effective range parameter. The correlated Gaussian expansion calculation in Ref. [13] found $\kappa r_{f d} \approx 0.08(1)$. Neutron-deuteron scattering data also favors a small value for $\kappa r_{f d}$. However the sign of $\kappa r_{f d}$ has remained an open question. In our continuum calculations presented here, we use the STM equation to calculate the scattering length and effective range parameter. We find the values $\kappa a_{f d}=1.17907(1)$ and $\kappa r_{f d}=-0.0383(3)$.

Our main focus though is to benchmark lattice calculations of the fermion-dimer scattering length and effective range parameter. For the lattice calculation we apply the finite- 
volume phase-shift analysis of Lüscher. We show that finite-volume topological corrections to the dimer binding energy must be considered in order to obtain accurate results. Once these topological corrections are included in the finite-volume analysis, we find that the lattice and continuum calculations are in full agreement. We use two different lattice Hamiltonian formulations, that agree in the continuum limit. As a final result we find:

$$
\kappa a_{f d}=1.174(9), \quad \kappa r_{f d}=-0.029(13)
$$

\section{NOTATION AND FORMALISM}

Few-body systems of two-component fermions with short-range interactions and large scattering lengths in comparison to interparticle distances show universal properties. Physics in such systems does not depend on the structure of the interactions at short distances. The problem of three two-component fermions at low energies can be described by a local quantum field theory whose only interaction term is a two-body contact interaction. In the following, we will always consider equal mass fermions with mass $m_{\uparrow}=m_{\downarrow}=m$. The extension to unequal masses is straightforward. The free non-relativistic effective Hamiltonian in momentum space can be written as

$$
H_{0}=\sum_{i=\uparrow, \downarrow} \int d^{3} \vec{p} \frac{\vec{p}^{2}}{2 m} a_{i}^{\dagger}(\vec{p}) a_{i}(\vec{p})
$$

where $a_{i}$ and $a_{i}^{\dagger}$ are annihilation and creation operators. In position space these operators can be expressed as

$$
a_{i}(\vec{r})=\frac{1}{(2 \pi)^{3}} \int d^{3} \vec{p} e^{i \vec{p} \cdot \vec{r}} a_{i}(\vec{p}), \quad a_{i}^{\dagger}(\vec{r})=\frac{1}{(2 \pi)^{3}} \int d^{3} \vec{p} e^{-i \vec{p} \cdot \vec{r}} a_{i}^{\dagger}(\vec{p}) .
$$

Combining Eqs. (2) and (3) the free Hamiltonian in configuration space is then given by

$$
\begin{aligned}
H_{0} & =-\frac{1}{2 m} \sum_{i=\uparrow, \downarrow} \int d^{3} \vec{r} a_{i}^{\dagger}(\vec{r}) \vec{\nabla}^{2} a_{i}(\vec{r}) \\
& =\frac{1}{2 m} \sum_{i=\uparrow, \downarrow} \int d^{3} \vec{r}\left(\vec{\nabla} a_{i}^{\dagger}(\vec{r})\right)\left(\vec{\nabla} a_{i}(\vec{r})\right) .
\end{aligned}
$$

Now we introduce an interaction between the fermions via the potential

$$
V\left(\vec{r}, \vec{r}^{\prime}\right)=\frac{1}{2} \sum_{i, j=\uparrow, \downarrow} \int d^{3} \vec{r} \int d^{3} \vec{r}^{\prime}: a_{i}^{\dagger}(\vec{r}) a_{i}(\vec{r}) \mathcal{V}\left(\vec{r}-\vec{r}^{\prime}\right) a_{j}^{\dagger}\left(\vec{r}^{\prime}\right) a_{j}\left(\vec{r}^{\prime}\right):
$$

where : ... : denotes normal ordering. At low energies, the potential (5) can be replaced by a delta-function interaction

$$
\mathcal{V}\left(\vec{r}-\vec{r}^{\prime}\right)=C_{0} \delta^{(3)}\left(\vec{r}-\vec{r}^{\prime}\right),
$$

and the the lowest order effective Hamiltonian for two-component fermions is

$$
H=-\frac{1}{2 m} \sum_{i=\uparrow, \downarrow} \int d^{3} \vec{r} a_{i}^{\dagger}(\vec{r}) \vec{\nabla}^{2} a_{i}(\vec{r})+C_{0} \int d^{3} \vec{r} a_{\uparrow}^{\dagger}(\vec{r}) a_{\uparrow}(\vec{r}) a_{\downarrow}^{\dagger}(\vec{r}) a_{\downarrow}(\vec{r}) .
$$


$C_{0}$ denotes the two-body coupling constant and is directly related to the fermion-fermion scattering length. It is assumed to be negative so that the interaction is attractive. The exact value of $C_{0}$ depends on the scheme used to regulate the short distance behavior.

In the next step we consider the two-body and three-body systems of two-component fermions in Hamiltonian lattice formalism and use the Lanczos method [20] to find the lowest eigenvalues. Further details of the Hamiltonian lattice formulation can be found in [21 23]. Let $\vec{n}$ denote spatial lattice points on a three-dimensional $L \times L \times L$ periodic cube. We use lattice units where physical quantities are multiplied by powers of the spatial lattice spacing $a_{\text {latt }}$ to make the combination dimensionless. The two-component fermions are labelled as spin-up and spin-down, the lattice annihilation operators are written as $a_{\uparrow}(\vec{n})$ and $a_{\downarrow}(\vec{n})$. The free non-relativistic Hamiltonian of two-component fermions with only short-range interaction corresponding to the Hamiltonian (44) on the three dimensional lattice is

$$
H_{0}=\frac{3}{m} \sum_{\vec{n}, i=\uparrow, \downarrow} a_{i}^{\dagger}(\vec{n}) a_{i}(\vec{n})-\frac{1}{2 m} \sum_{\hat{\mu}=\hat{1}, \hat{2}, \hat{3} \vec{n}, i=\uparrow, \downarrow}\left[a_{i}^{\dagger}(\vec{n}) a_{i}(\vec{n}+\hat{\mu})+a_{i}^{\dagger}(\vec{n}) a_{i}(\vec{n}-\hat{\mu})\right],
$$

where $\hat{\mu}$ is the spatial lattice unit vector. We define the spin-density operators

$$
\begin{aligned}
& \rho_{\uparrow}(\vec{n})=a_{\uparrow}^{\dagger}(\vec{n}) a_{\uparrow}(\vec{n}) \\
& \rho_{\downarrow}(\vec{n})=a_{\downarrow}^{\dagger}(\vec{n}) a_{\downarrow}(\vec{n}),
\end{aligned}
$$

and consider two different kinds of Hamiltonians. In the first Hamiltonian we have only a single-site interaction. This Hamiltonian is

$$
H_{1}=H_{0}+C_{1} \sum_{\vec{n}} \rho_{\uparrow}(\vec{n}) \rho_{\downarrow}(\vec{n}) .
$$

We consider a second Hamiltonian using a contact interaction as well as nearest-neighbour interaction terms in order to eliminate the two-body effective range parameter,

$$
\begin{aligned}
H_{2} & =H_{0}+C_{2} \sum_{\vec{n}} \rho_{\uparrow}(\vec{n}) \rho_{\downarrow}(\vec{n}) \\
& +C_{2}^{\prime} \sum_{\hat{\mu}=\hat{1}, \hat{2}, \hat{3}} \sum_{\vec{n}}\left[\rho_{\uparrow}(\vec{n}) \rho_{\downarrow}(\vec{n}+\hat{\mu})+\rho_{\uparrow}(\vec{n}+\hat{\mu}) \rho_{\downarrow}(\vec{n})\right] .
\end{aligned}
$$

The finite lattice spacing error in these two Hamiltonians is of order $a_{\text {latt }}^{2}$.

The next step is to determine the interaction coefficients, $C_{1}, C_{2}$ and $C_{2}^{\prime}$ using Lüscher's formula [24 26]. At present Lüscher's formula is a standard tool in lattice quantum chromodynamics and in lattice effective field theory. It relates the two-body energy levels in a finite volume to the S-wave phase shift.

$$
p \cot \delta_{0}(p)=\frac{1}{\pi L} S(\eta), \quad \eta=\left(\frac{L p}{2 \pi}\right)^{2},
$$

where $S(\eta)$ is the three-dimensional zeta-function, $L$ is the length of the box and $p$ is the center-of-mass momentum. The zeta-function in three dimensions is defined as

$$
S(\eta)=\lim _{\Lambda \rightarrow \infty}\left[\sum_{\vec{n}} \frac{\theta\left(\Lambda^{2}-\vec{n}^{2}\right)}{\vec{n}^{2}-\eta}-4 \pi \Lambda\right] .
$$


For $|\eta|<1$ we can expand $S(\eta)$ in powers of $\eta$,

$$
\begin{aligned}
S(\eta) & =-\frac{1}{\eta}+\lim _{\Lambda \rightarrow \infty}\left[\sum_{\vec{n} \neq 0} \frac{\theta\left(\Lambda^{2}-\vec{n}^{2}\right)}{\vec{n}^{2}-\eta}-4 \pi \Lambda\right], \\
& =-\frac{1}{\eta}+S_{0}+S_{1} \eta+S_{2} \eta^{2}+S_{3} \eta^{3}+\cdots
\end{aligned}
$$

where

$$
S_{0}=\lim _{\Lambda \rightarrow \infty}\left[\sum_{\vec{n} \neq 0} \frac{\theta\left(\Lambda^{2}-\vec{n}^{2}\right)}{\vec{n}^{2}}-4 \pi \Lambda\right], \quad S_{i}=\sum_{\vec{n} \neq 0} \frac{1}{\left(\vec{n}^{2}\right)^{i+1}} .
$$

The first few coefficients are

$$
\begin{aligned}
& S_{0}=-8.913631, \quad S_{1}=16.532288, \quad S_{2}=8.401924, \quad S_{3}=6.945808 \\
& S_{4}=6.426119, \quad S_{5}=6.202149, \quad S_{6}=6.098184, \quad S_{7}=6.048263 .
\end{aligned}
$$

Lüscher's formula does not include the contribution from higher partial waves but at asymptotically small momenta we can neglect such corrections. For small momenta we have the effective range expansion,

$$
p \cot \delta_{0}(p) \simeq-\frac{1}{a_{s}}+\frac{1}{2} r_{0} p^{2}+\cdots
$$

where $a_{s}$ is the scattering length and $r_{0}$ is the effective range. Thus the effective range parameters can be extracted from the finite volume energy levels using Eq. (13). In terms of $\eta$, the energy of the dimer is

$$
E=\frac{p^{2}}{m}=\frac{\eta}{m}\left(\frac{2 \pi}{L}\right)^{2}
$$

The interaction coefficient $C_{1}$ is tuned to construct two-body binding states (dimers) comprised of one spin-up and one spin-down fermion of energies $-1.5 \mathrm{MeV},-2.0 \mathrm{MeV}$, $-2.5 \mathrm{MeV},-3.0 \mathrm{MeV},-3.5 \mathrm{MeV}$ and $-4.0 \mathrm{MeV}$ in the large volume $(L=80)$ using the Lanczos method. In such a large volume the finite volume corrections to the dimer binding energy are negligible. In our calculation we take $m=939 \mathrm{MeV}$ and $a_{\text {latt }}^{-1}=100 \mathrm{MeV}$. To find the interaction coefficients of lattice Hamiltonian $\mathrm{H}_{2}$ we proceed as follows. Setting the effective range to zero requires that the following relation should be satisfied near threshold

$$
\frac{1}{\pi L} S(\eta)=p \cot \delta_{0}(p) \simeq-\frac{1}{a_{s}}+O\left(p^{4}\right)
$$

The interaction coefficients $C_{2}$ and $C_{2}^{\prime}$ are tuned in order to give the binding energies listed above for the ground state in a large volume $(L=80)$ and to fullfil Eq. (20) for the first excited state. The plot for $p \cot \delta_{0}(p)$ versus $p^{2}$ for the first excited state is shown in Fig. 1. The different values of $p$ are generated by calculating for different box sizes. We note that $p \cot \delta_{0}(p)$ has zero slope near threshold since we set the effective range to zero. Both Hamiltonians reproduce the same continuum limit of fermions with attractive zero-range interactions. The corresponding values for the interaction coefficients are summarized in Table [1. 


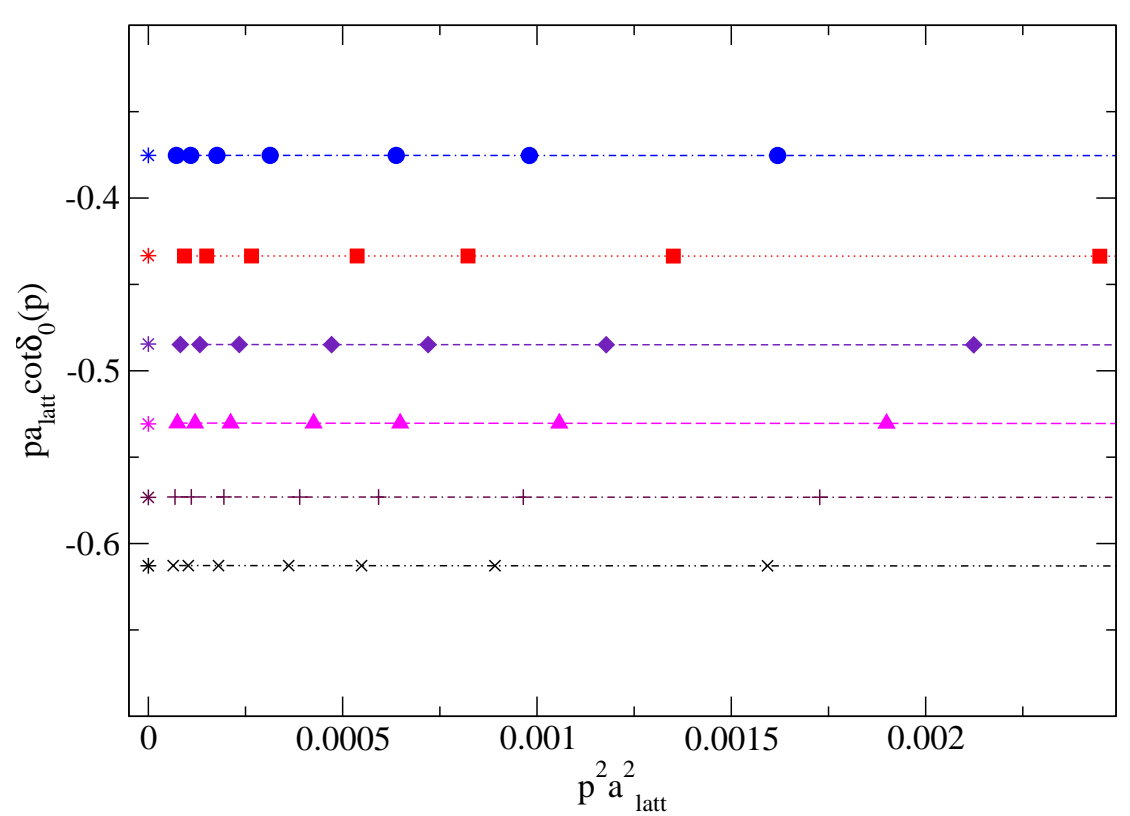

FIG. 1: Plot of $p \cot \delta_{0}(p)$ versus $p^{2}$ for the lattice Hamiltonian $H_{2} \cdot \bullet, \mathbf{a}, \bullet, \mathbf{\Delta},+, \times$, represent data points corresponding to the bound states of energies $-1.5 \mathrm{MeV},-2.0 \mathrm{MeV},-2.5 \mathrm{MeV}$, $-3.0 \mathrm{MeV},-3.5 \mathrm{MeV}$ and $-4.0 \mathrm{MeV}$, respectively. * denotes $p \cot \delta_{0}(p)$ at the zero momentum limit.

TABLE I: The values of interaction coefficients for the six considered dimers. All quantities are given in units of the lattice spacing $a_{\text {latt }}=(100 \mathrm{MeV})^{-1}$.

\begin{tabular}{cccc}
\hline \hline$E^{d}$ & $m C_{1}$ & $m C_{2}$ & $m C_{2}^{\prime}$ \\
\hline-0.015 & -4.51091 & -4.34554 & -0.30082 \\
-0.020 & -4.61299 & -4.45733 & -0.34273 \\
-0.025 & -4.70675 & -4.55883 & -0.34658 \\
-0.030 & -4.79466 & -4.64749 & -0.36339 \\
-0.035 & -4.87817 & -4.73801 & -0.36452 \\
-0.040 & -4.95823 & -4.82374 & -0.36696 \\
\hline
\end{tabular}

We use the interaction coefficients in Table \and diagonalize both Hamiltonians (11) and (12) utilizing the Lanczos method to determine the ground state energy at rest for the six considered dimers in the periodic volumes $L^{3}$ ranging from $L=6$ to $L=17$. We also use the same interaction coefficients and diagonalization method to find the ground state energy of the fermion-dimer systems. These energies are summarized in the tables in Appendix A.

Now we turn our attention to the fermion-dimer scattering in a periodic cube. It is known that there are exponentially small corrections to the scattering energy of the fermion-dimer system at finite volume due to range effects. We can remove this error by extrapolation to infinite volume. However there is another error which is independent of the fermion-dimer scattering process, namely the finite volume error in the dimer binding energy. One might think that this error can be removed by substracting the dimer binding energy from the total energy of the fermion-dimer scattering system. But this is not quite correct since we 
calculate the scattering process in the center-of-mass frame and therefore the dimer has some recoil momentum. The corrections to the dimer binding energy in the moving frame differ from its rest frame due to the topological phases for the moving dimer in the finite volume [2, 27].

\section{BOUND STATE IN A MOVING FRAME}

Lüscher derived the finite-volume corrections to the binding energy of two-body bound states for interactions with finite range [24]. The shift in the energy of a bound state in a periodic cube at rest is given by

$$
\Delta E_{\overrightarrow{0}}(L) \simeq \sum_{|\vec{n}|=1} \int d^{3} r \phi_{\infty}^{*}(\vec{r}) V(\vec{r}) \phi_{\infty}(\vec{r}+\vec{n} L),
$$

where $\phi_{\infty}$ is the infinite-volume wavefunction as a function of the relative separation $\vec{r}$ and $V(\vec{r})$ is the interaction potential. Using a Galilean transformation we can find the wavefunction of the bound state in a periodic cube of length $L$ moving with momentum $2 \pi \vec{k} / L$ for integer $\vec{k}$. This wavefunction in a periodic cube has a phase dependence which can be factorized out,

$$
\phi_{L}(\vec{r}+\vec{n} L)=e^{-2 i \pi \alpha \vec{k} \cdot \vec{n}} \phi_{L}(\vec{r}),
$$

where $\alpha=m_{\uparrow} /\left(m_{\uparrow}+m_{\downarrow}\right)$ for the general case of unequal masses and $\vec{n}$ is an integer. Each phase twist at the boundaries induces a shift in the binding energy of the dimer. Using Eqs. (21) and (22) for the $S$-wave bound state, the finite volume correction in a moving frame is

$$
\Delta E_{\vec{k}}(L) \approx \tau(\vec{k}, \alpha) \Delta E_{\overrightarrow{0}}(L)
$$

where

$$
\tau(\vec{k}, \alpha)=\frac{1}{3} \sum_{i=1}^{3} \cos \left(2 \pi \alpha k_{i}\right),
$$

and $\Delta E_{\vec{k}}(L)$ and $\Delta E_{\overrightarrow{0}}(L)$ represent finite volume correction to the binding energy of the dimer in the moving and rest frame, respectively.

These corrections have a universal dependence on momentum determined by the number and mass of the constituents. In asymptotically large volumes the corrections are exponentially small and can be neglected. But if the volume is relatively small, this shift can be comparable to that of the scattering process of the fermion and dimer.

In order to calculate finite-volume corrections due to the binding energy of the dimer in the total scattering energy of the fermion-dimer system, let $E^{f d}(p, L)$ be the total scattering energy with radial momentum $p$ and $E_{\vec{k}}^{d}(L)$ the finite volume energy due to binding for the dimer with momentum $2 \pi \vec{k} / L$ in a periodic cube of length $L$. In the asymptotic limit $L \rightarrow \infty$, with $p$ scaling as $1 / L$, we can neglect the mixing with higher-order singular solutions to the Helmholtz equation. For S-wave scattering of a dimer and a fermion with radial momentum $p$ and separation $\vec{r}$ between the center of mass of the dimer and the fermion, the position-space scattering wavefunction is

$$
\left\langle\vec{r} \mid \Psi_{p}\right\rangle=c \sum_{\vec{k}} \frac{e^{\frac{2 i \pi \vec{k} \cdot \vec{r}}{L}}}{(2 \pi \vec{k} / L)^{2}-p^{2}}
$$


TABLE II: Toplogical factor for six considered dimers. The upper part of table calculated by using the ground state energies corresponding to $H_{1}$ and the lower part by using the ground state energies corresponding to $\mathrm{H}_{2}$.

\begin{tabular}{|c|c|c|c|c|c|c|c|c|c|c|c|c|}
\hline $\bar{L}$ & 17 & 16 & 15 & 14 & 13 & 12 & 11 & 10 & 9 & 8 & 7 & 6 \\
\hline$\tau_{d}^{1.5}$ & 0.84351 & 0.81836 & 0.78862 & 0.75386 & 0.71392 & 0.66915 & 0.62052 & 0.56968 & - & - & - & - \\
\hline$\tau_{d}^{2.0}$ & 0.89079 & 0.87205 & 0.84912 & 0.82108 & 0.78709 & 0.74648 & 0.69916 & 0.64592 & 0.58872 & - & - & - \\
\hline$\tau_{d}^{2.5}$ & 0.91790 & 0.90351 & 0.88565 & 0.86323 & 0.83517 & 0.80025 & 0.75743 & 0.70632 & 0.64778 & 0.58452 & - & - \\
\hline$\tau_{d}^{3.0}$ & 0.93511 & 0.92349 & 0.90912 & 0.89090 & 0.86769 & 0.83803 & 0.80038 & 0.75340 & 0.69669 & 0.63176 & 0.56267 & - \\
\hline$\tau_{d}^{3.5}$ & 0.94645 & 0.93706 & 0.92520 & 0.91009 & 0.89060 & 0.86532 & 0.83248 & 0.79022 & 0.73731 & 0.67442 & 0.60554 & - \\
\hline$\tau_{d}^{4.0}$ & 0.95466 & 0.94675 & 0.93676 & 0.92396 & 0.90735 & 0.88560 & 0.85681 & 0.81889 & 0.76973 & 0.70858 & 0.63804 & 0.56535 \\
\hline$\tau_{d}^{1.5}$ & 0.82960 & 0.80247 & 0.77064 & 0.73384 & 0.69221 & 0.64649 & 0.59810 & 0.54894 & - & - & - & - \\
\hline$\tau_{d}^{2.0}$ & 0.88030 & 0.85976 & 0.83471 & 0.80429 & 0.76775 & 0.72470 & 0.67555 & 0.62176 & 0.56594 & - & - & - \\
\hline$\tau_{d}^{2.5}$ & 0.90967 & 0.89377 & 0.87401 & 0.84938 & 0.81873 & 0.78093 & 0.73524 & 0.68189 & 0.62272 & 0.56130 & - & - \\
\hline$\tau_{d}^{3.0}$ & 0.92804 & 0.91529 & 0.89930 & 0.87907 & 0.85338 & 0.82077 & 0.77979 & 0.72952 & 0.67045 & 0.60542 & 0.53965 & - \\
\hline$\tau_{d}^{3.5}$ & 0.94077 & 0.93030 & 0.91709 & 0.90025 & 0.87859 & 0.85060 & 0.81446 & 0.76847 & 0.71181 & 0.64593 & 0.57567 & - \\
\hline$\tau_{d}^{4.0}$ & 0.94988 & 0.94107 & 0.92992 & 0.91564 & 0.89714 & 0.87294 & 0.84112 & 0.79953 & 0.74636 & 0.68156 & 0.60882 & 0.53618 \\
\hline
\end{tabular}

with some normalization constant $c$. The total energy $E^{f d}(p, L)$ is given by

$$
E^{f d}(p, L)=\frac{\left\langle\Psi_{p}|H| \Psi_{p}\right\rangle}{\left\langle\Psi_{p} \mid \Psi_{p}\right\rangle}=\frac{1}{\mathcal{N}} \sum_{\vec{k}} \frac{\frac{p^{2}}{m}+E_{\vec{k}}^{d}(L)}{\left(\vec{k}^{2}-\eta\right)^{2}}
$$

where $\mathcal{N}=\sum_{\vec{k}}\left(\vec{k}^{2}-\eta\right)^{-2}$. The finite-volume correction due to the binding energy of the dimer in the scattering process is

$$
E^{f d}(p, L)-E^{f d}(p, \infty)=\tau_{d}(\eta) \Delta E_{\overrightarrow{0}}^{d}(L),
$$

where the topological volume factor for $m_{\uparrow}=m_{\downarrow}=m$ is given by

$$
\tau_{d}(\eta)=\frac{1}{\mathcal{N}} \sum_{\vec{k}} \frac{\tau\left(\vec{k}, \frac{1}{2}\right)}{\left(\vec{k}^{2}-\eta\right)^{2}}
$$

We find $\tau_{d}(\eta)$ iteratively. We determine $p^{2}$ and $\eta$ by subtracting the ground state energy of the dimer in the rest frame from the total energy. Using this $\eta$ and Eq. (28) we find $\tau_{d}(\eta)$ and use it to modify the binding energy of the dimer. We repeat this process until $\eta$ does not change anymore. For very large box length $(L=2000)$ we achieve a fixed point after three iterations. The ratio of the finite volume corrections in the moving and rest frame are collected in Table II. We note that this ratio is significantly smaller than one at small volumes. For large volumes this ratio can be neglected. The binding energies of the dimers in the boosted frame are summarized in Appendix A. We emphasize that the finite-volume correction in Eq. (27) has nothing to do with the interaction between dimer and fermion and should therefore be subtracted from the total energy before using Lüscher's scattering relation. This subtraction reduces systematic errors in lattice calculations. We note that in the total scattering energy there are also corrections corresponding to the scattering process which we will remove by extrapolation to the infinite volume and to the continuum limit. 

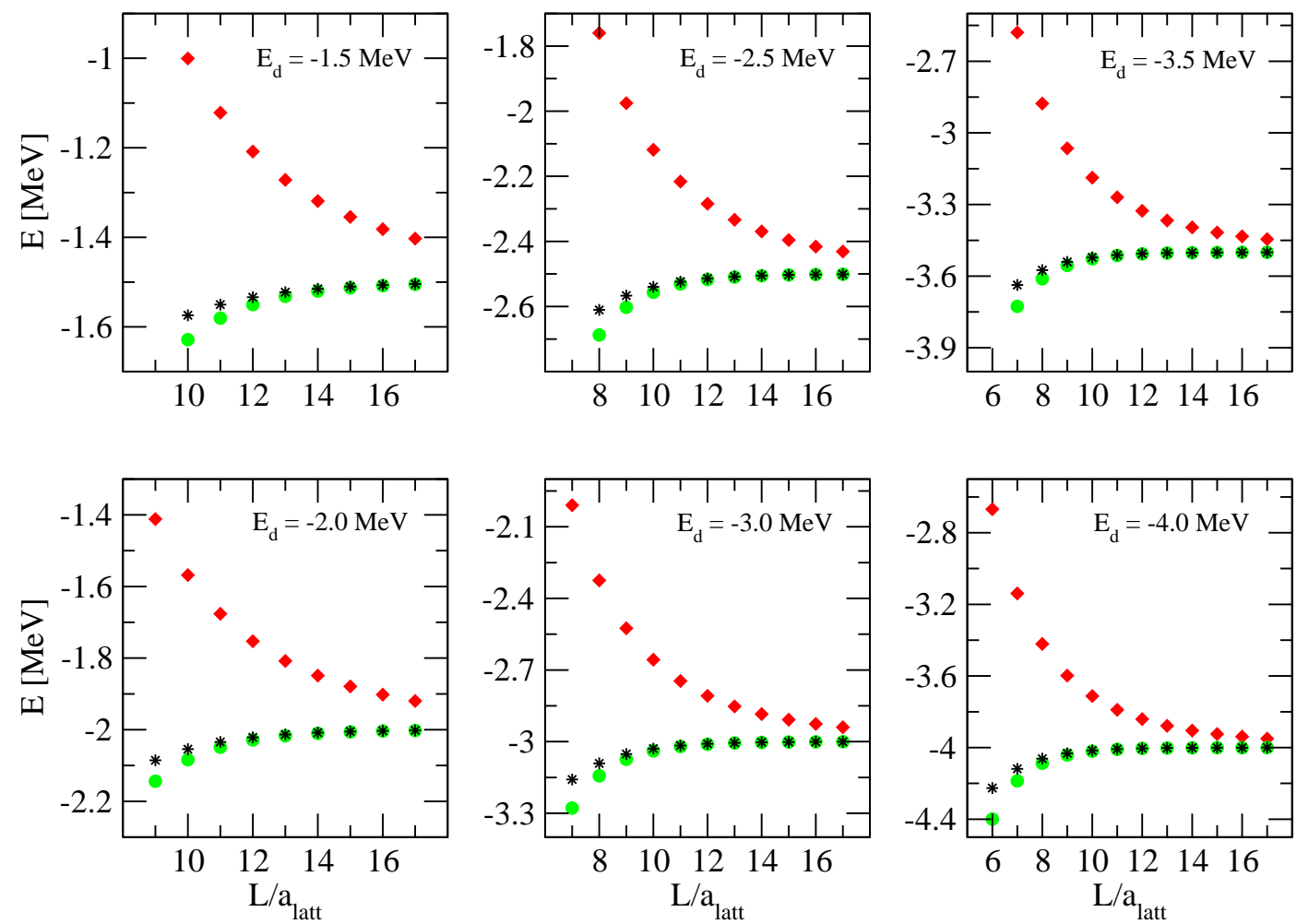

FIG. 2: Plots of ground state energies corresponding to the lattice Hamiltonian $H_{1}$ versus $L$. Circle and star represent the ground state energies of the dimers in the rest- and boosted-frame respectively and diamond stands for ground state energies of the fermion-dimer system.

\section{FERMION-DIMER SCATTERING}

\section{A. Lattice Calculation}

In order to find the radial momentum, $p$, in the fermion-dimer systems, we subtract the binding energies of the dimers in the moving frame from the total scattering energies of the fermion-dimer systems. The ground state energies of the six considered dimers in the rest and moving frame and the ground state energies of the fermion-dimer system for both lattice Hamiltonians, $H_{1}$ and $H_{2}$, are shown in Figs. 2 and 3. As discussed above, the difference between the ground state energies of the dimers in the rest and moving frame is bigger in small volumes. In this case it is comparable to the corrections due to the scattering process.

The difference $\Delta E(L)$ is the kinetic energy of the fermion-dimer system. We use this to find the radial momentum $p$. In a naive calculation, we take $\tau_{d}(\eta)$ equal to one and thus implicitly assume the corrections to the binding energy of the dimer the in rest and moving frame are equal. We subtract the binding energy of the dimer in the rest frame from the total energy of the fermion-dimer system in order to find $\Delta E(L)$ and eliminate the correction due to the dimer binding energy in finite volume,

$$
\Delta E^{\text {naive }}(L)=\Delta E^{f d}(L)-\Delta E_{\overrightarrow{0}}^{d}(L) .
$$

In the full calculation, we subtract the binding energy of the dimer in the boosted frame in order to determine $\Delta E(L)$ and eliminate the finite volume correction corresponding to the 

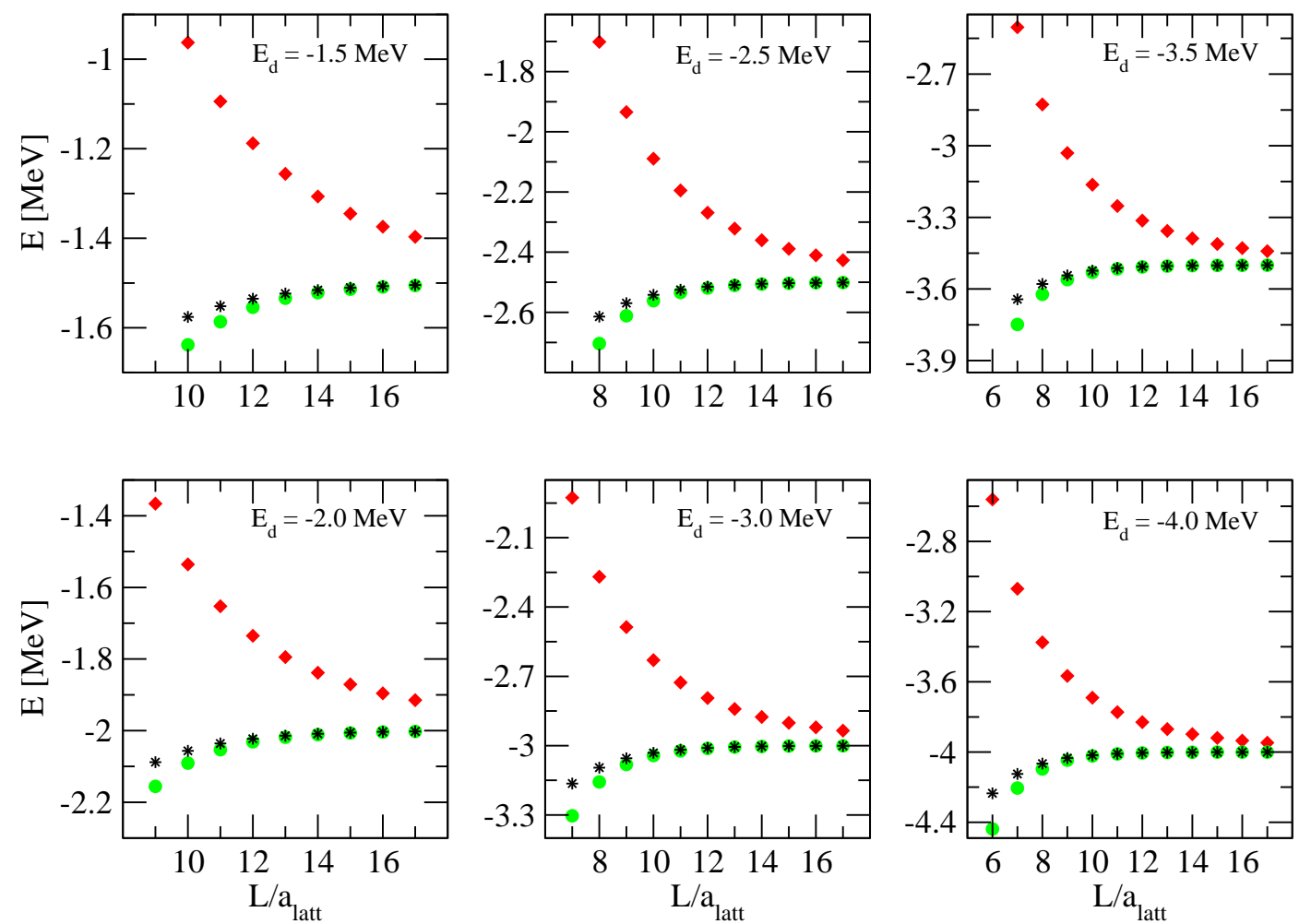

FIG. 3: Plots of ground state energies corresponding to the lattice Hamiltonian $\mathrm{H}_{2}$ versus $L$. Circle and star represent the ground state energies of the dimers in the rest- and boosted-frame respectively and diamond stands for ground state energies of the fermion-dimer system.

binding energy of the dimer,

$$
\begin{aligned}
\Delta E^{f u l l}(L) & =\Delta E^{f d}(L)-\Delta E_{\vec{k}}^{d}(L) \\
& =\Delta E^{f d}(L)-\tau_{d}(\eta) \Delta E_{\overrightarrow{0}}^{d}(L) .
\end{aligned}
$$

After subtracting the corrections corresponding to the binding energy of the dimer from the total energy we use the Lüscher's formula with this energy and calculate $p \cot \delta_{0}(p)$ for six different lattice spacings. We also calculate $p \cot \delta_{0}(p)$ for the case in which we subtracted only the binding energy of the dimer at rest frame from the total energy. This simply means we replace $\tau_{d}(\eta)$ by 1 . In Fig. 4 , the results are plotted versus $p^{2}$. To extrapolate to the infinite volume we fit a polynomial of second order to the data points. We write this results as dimensionless combinations multiplied by powers of the dimer binding momentum $\kappa$. By comparing the naive calculation plots in Fig. 4 (a) and (c) with the full calculation plots (b) and (d), we clearly see the effect of the topological phase factor. This correction is quite large for scattering in smaller volumes. The change in slope in plot (d) compared to plot (b) is expected since we tuned the effective range of interaction to zero for $H_{2}$. From these results, we determine the low-energy parameters for fermion-dimer scattering and extrapolate to the continuum limit. 

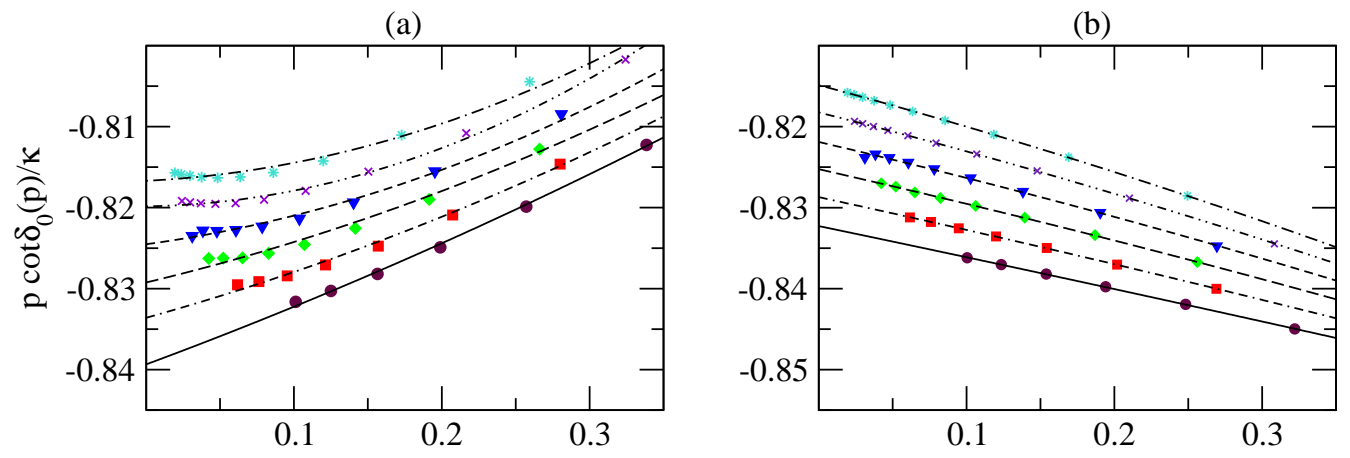

(c)
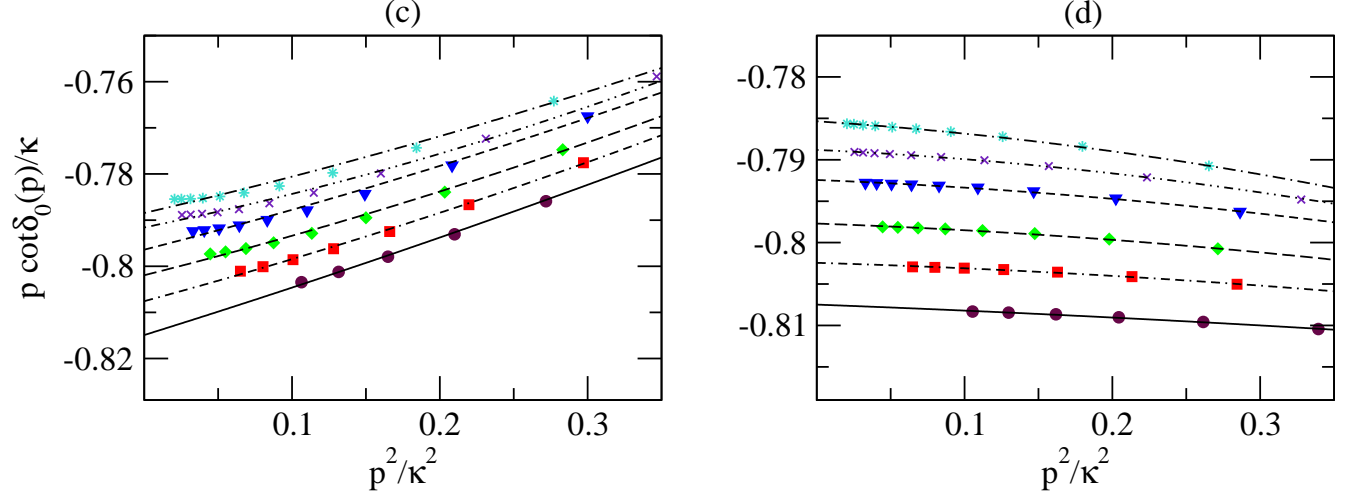

FIG. 4: Plots of $p \cot \delta_{0}(p)$ versus $p^{2}$. (a) Naive calculation for $H_{1}$. (b) Full calculation for $H_{1}$. (c) Naive calculation for $H_{2}$. (d) Full calculation for $H_{2}$. $\bullet, \mathbf{\square}, \mathbf{\nabla}, \times$ and $*$ represent data points corresponding to the bound states of energies $-1.5 \mathrm{MeV},-2.0 \mathrm{MeV},-2.5 \mathrm{MeV},-3.0 \mathrm{MeV}$, $-3.5 \mathrm{MeV}$ and $-4.0 \mathrm{MeV}$ respectively.

\section{B. STM Equation}

The STM equation for the S-wave fermion-dimer scattering amplitude $\mathcal{T}_{f d}(k, p ; E)$ can be written as [18, 19]

$$
\mathcal{T}_{f d}(k, p ; E)=-\frac{8 \kappa}{3} M(k, p ; E)-\frac{2}{\pi} \int_{0}^{\infty} \frac{d q q^{2} M(q, p ; E) \mathcal{T}_{f d}(k, q ; E)}{-\kappa+\sqrt{3 q^{2} / 4-m E-i \epsilon}}
$$

where $k$ and $p$ are the incoming and outgoing momenta of the fermion and dimer in the center-of-mass frame, $\kappa$ is the binding momentum of the dimer, and

$$
E=\frac{3}{4} \frac{p^{2}}{m}-\frac{\kappa^{2}}{m}
$$

is the total energy. The inhomogeneous term

$$
M(k, p ; E)=\frac{1}{2 p k} \ln \left(\frac{p^{2}+p k+k^{2}-m E}{p^{2}-p k+k^{2}-m E}\right),
$$



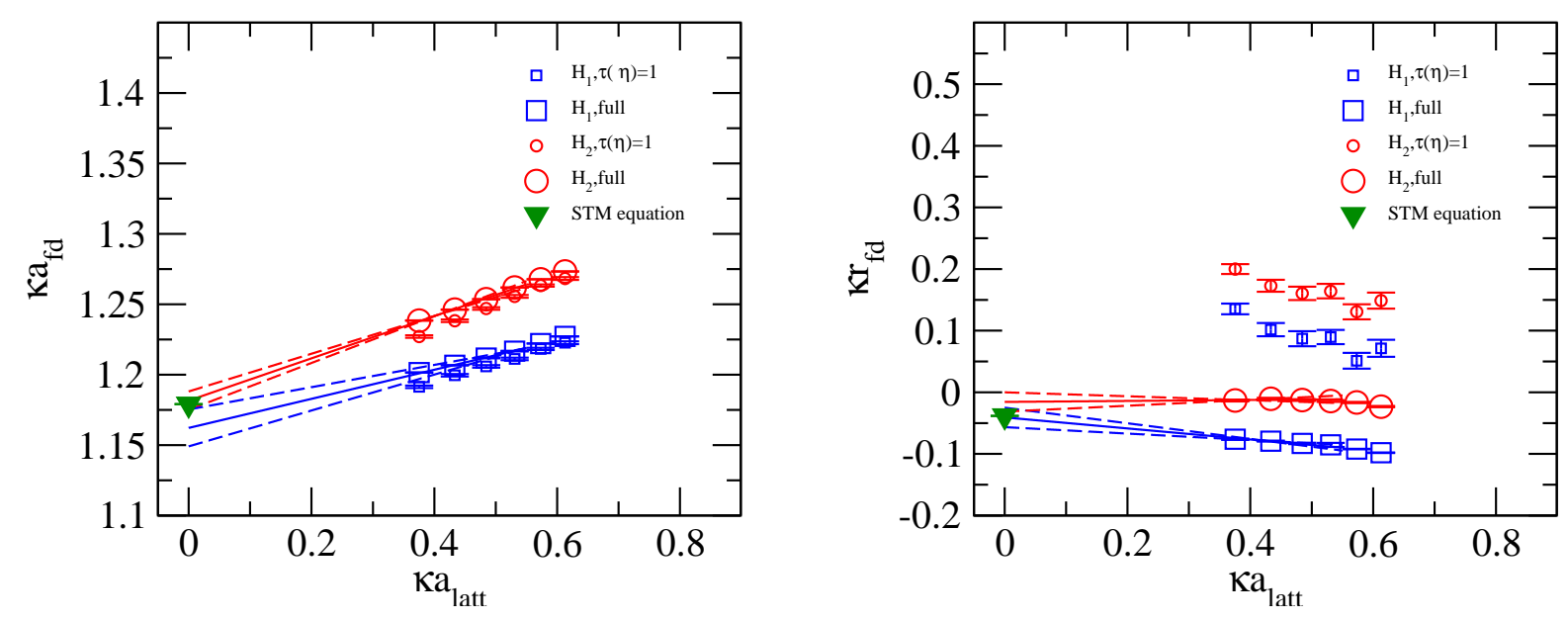

FIG. 5: Left: Lattice results and continuum extrapolation with error estimates for the fermiondimer scattering length. Right: Lattice results and continuum extrapolation with error estimates for the fermion-dimer effective range. For comparison we show the continuum result obtain via the Skorniakov-Ter-Martirosian equation. The error bands show statistical errors corresponding to the fitting procedure shown in Fig. 4

is given by the S-wave projected one-fermion exchange. The fermion-dimer scattering phase shifts are obtained by evaluating Eq. (31) at the on-shell point:

$$
\mathcal{T}_{f d}(p, p ; E)=\frac{1}{p \cot \delta_{0}(p)-i p} .
$$

By discretizing the momenta $p$ and $k$ the STM equation (31) can be transformed into a matrix equation which can be solved numerically. The numerical errors in the solution of this equation are negligible. The effective range parameters are extracted from the scattering amplitude by fitting a polynomial in $p^{2}$ to $1 / \mathcal{T}_{f d}(p, p ; E)+i p$. Errors are estimated by varying the degree of the fitted polynomial.

\section{COMPARARISON OF RESULTS AND DISCUSSION}

From the lattice calculation of the phase shifts in Fig. 4 we can extract the effective range parameters. Our results for the scattering length, $a_{f d}$, and the effective range parameter, $r_{f d}$, are shown in Fig. 5. We analyze only the plots in Fig. 4 (b) and (d) which contain the full calculations corresponding to $H_{1}$ and $H_{2}$, respectively. By fitting a polynomial of second order to each set of data we find a scattering length and a effective range in infinite volume for both lattice Hamiltonians. These data points are plotted in Fig 5. In order to extrapolate to the continuum limit $a_{\text {latt }} \rightarrow 0$, we use a linear function. The results for the low-energy parameters that we get for these two independent representations of the lattice Hamiltonians are

$$
\begin{aligned}
& \kappa a_{f d}=1.162(13), \quad \kappa r_{f d}=-0.041(16) \quad \text { for } H_{1} \\
& \kappa a_{f d}=1.181(7), \quad \kappa r_{f d}=-0.016(16) \quad \text { for } H_{2} .
\end{aligned}
$$

To extrapolate to the continuum limit in the lattice Hamiltonian calculations we used

only the data points corresponding to the four smallest lattice spacings. For the other data 
points, the Compton wavelength of the bound state is comparable to the lattice spacing. We estimate the systematic errors in the continuum extrapolation of the fermion-dimer scattering length and effective range by extrapolation to the continuum limit using only the first two data points and taking the interval between these extrapolation values and the central values obtained using all four data points as the systematic errors. The agreement between these two independent calculations is consistent with our estimate of the systematic errors. As we see from Fig. [5 the inclusion of the topological volume factor $\tau_{d}(\eta)$ improves the accuracy, especially in the calculation of the effective range parameter. With a very conservative estimation of the systematic error we are able to say that the value of the the fermion-dimer scattering length in units of the dimer binding momentum is in between 1.149 and 1.188. The value of fermion-dimer effective range in units of the dimer binding momentum is between zero and -0.057 . Our final result is given by the weighted averages of the values in Eq. (35) and (36):

$$
\kappa a_{f d}=1.174(9), \quad \kappa r_{f d}=-0.029(13) .
$$

In calculating the average, we assumed that the statistical probability distribution of the measured variables are Gaussian and independent of each other. Using standard error propagation, we find the uncertainty in the average values. Our results (37) are in excellent agreement with the continuum calculation using the STM integral equation (31):

$$
\kappa a_{f d}=1.17907(1), \quad \kappa r_{f d}=-0.0383(3) .
$$

\section{OUTLOOK}

We have presented benchmark calculations for fermion-dimer scattering using a Hamiltonian lattice formalism and in the continuum using the STM integral equation. We obtain excellent agreement between both approaches. The finite-volume lattice methods presented here can be applied to ab initio calculations for elastic scattering of nuclei, cold atoms, and hadronic molecules. Of particular interest in nuclear physics are calculations of the lowenergy scattering of neutrons upon nuclei. Soft neutron scattering upon nuclei is relevant to the design of container materials used in ultracold neutron experiments. They are also an important probe of the properties of nuclei surrounded by a dilute superfluid neutron gas. It is widely believed that this physical situation with nuclei in a neutron gas is realized in the inner crust of neutron stars.

Calculations of deuteron-neutron scattering in the spin-quartet channel should yield similar results to the idealized zero-range limit presented here. There will, however, be small corrections due to the range of the interactions as well as spin-dependent forces. Our methods can also be applied to neutron-deuteron scattering in the spin-doublet channel. Here there is an interesting connection with Efimov trimer physics in a finite volume [28 30]. As a first step in this direction, boson-dimer scattering for three identical bosons in the zero-range limit is currently being investigated [31].

There are also some very useful applications of the topological volume factor for binding energy calculations. By choosing different values of the center of mass motion, one can make the sum over topological volume factors vanish. This can be used to remove the leading and even subleading finite-volume corrections to the binding energy of two-body bound states. This technique is being pursued in a number of recent lattice QCD studies for the deuteron and other dibaryonic systems [27, 32]. 


\section{Acknowledgments}

We thank Dörte Blume and Martin Savage for useful discussions. Partial financial support from the Deutsche Forschungsgemeinschaft (SFB/TR 16), Helmholtz Association (contract number VH-VI-231), BMBF (grant 06BN9006), and U.S. Department of Energy (DE-FG02- 03ER41260) are acknowledged. This work was further supported by the EU HadronPhysics3 project "Study of strongly interacting matter".

\section{Appendix A: Ground State Energies}

TABLE III: Ground state energy of the dimers in the rest frame calculated by using $H_{1}$.

\begin{tabular}{|c|c|c|c|c|c|c|c|c|c|c|c|c|}
\hline$L$ & 17 & 16 & 15 & 14 & 13 & 12 & 11 & 10 & 9 & 8 & 7 & 6 \\
\hline$E_{\overrightarrow{0}}^{1.5}$ & -1.5051 & -1.5080 & -1.5126 & -1.5200 & -1.5316 & -1.5504 & -1.5804 & -1.6287 & $\begin{array}{c}- \\
-\end{array}$ & - & - & - \\
\hline$E_{\overrightarrow{0}}^{2.0}$ & -2.0022 & -2.0036 & -2.0060 & -2.0101 & -2.0170 & -2.0287 & -2.0489 & -2.0838 & -2.1439 & - & - & - \\
\hline$E_{\overrightarrow{0}}^{2.5}$ & -2.5010 & -2.5018 & -2.5031 & -2.50550 & -2.5097 & -2.5173 & -2.5311 & -2.5562 & -2.6024 & -2.6874 & - & - \\
\hline$E_{\overrightarrow{0}}^{3.0}$ & -3.0005 & -3.0009 & -3.0017 & -3.0032 & -3.0059 & -3.0109 & -3.0205 & -3.0388 & -3.0743 & -3.1434 & -3.2782 & - \\
\hline$E_{\overrightarrow{0}}^{3.5}$ & -3.5003 & -3.5005 & -3.5010 & -3.5019 & -3.5037 & -3.5071 & -3.5139 & -3.5275 & -3.5550 & -3.6112 & -3.7266 & - \\
\hline$E_{\overrightarrow{0}}^{4.0}$ & -4.0002 & -4.0003 & -4.0006 & -4.0012 & -4.0024 & -4.00 & -4.0097 & -4.0200 & -4.0415 & -4.0874 & -4.1861 & -4.3994 \\
\hline
\end{tabular}

TABLE IV: Ground state energy of the dimers in the rest frame calculated by using $H_{2}$.

\begin{tabular}{|c|c|c|c|c|c|c|c|c|c|c|c|c|}
\hline$L$ & 17 & 16 & 15 & 14 & 13 & 12 & 11 & 10 & 9 & 8 & 7 & 6 \\
\hline$E_{\overrightarrow{0}}^{1.5}$ & -1.5055 & -1.5087 & -1.5136 & -1.5215 & -1.5341 & -1.5543 & -1.5865 & -1.6382 & - & - & - & - \\
\hline$E_{\overrightarrow{0}}^{2.0}$ & -2.0024 & -2.0040 & -2.0066 & -2.0110 & -2.0185 & -2.0313 & -2.0532 & -2.0908 & -2.1556 & - & - & - \\
\hline$E_{\overrightarrow{0}}^{2.5}$ & -2.5011 & -2.5020 & -2.5034 & -2.5060 & -2.5106 & -2.5189 & -2.5339 & -2.5613 & -2.6115 & -2.7035 & - & - \\
\hline$E_{\overrightarrow{0}}^{3.0}$ & -3.0006 & -3.0011 & -3.0020 & -3.0035 & -3.0065 & -3.0121 & -3.0226 & -3.0428 & -3.0817 & -3.1573 & -3.3039 & - \\
\hline$E_{\overrightarrow{0}}^{3.5}$ & -3.5003 & -3.5006 & -3.5011 & -3.5021 & -3.5041 & -3.5079 & -3.5154 & -3.5304 & -3.5608 & -3.6225 & -3.7487 & - \\
\hline$E_{\overrightarrow{0}}^{4.0}$ & -4.0004 & -4.0005 & -4.0009 & -4.0015 & -4.0028 & -4.0055 & -4.0110 & -4.0223 & -4.0462 & -4.0968 & -4.2052 & -4.4384 \\
\hline
\end{tabular}

TABLE V: Ground state energy of fermion-dimer systems calculated by using $H_{1}$.

\begin{tabular}{|c|c|c|c|c|c|c|c|c|c|c|c|c|}
\hline$L$ & 17 & 16 & 15 & 14 & 13 & 12 & 11 & 10 & 9 & 8 & 7 & 6 \\
\hline$E_{1.5}^{f d}$ & -1.4029 & -1.3817 & -1.3545 & -1.3189 & -1.2718 & -1.2084 & -1.1216 & -1.0003 & - & - & - & - \\
\hline$E_{2.0}^{f d}$ & -1.9199 & -1.9022 & -1.8791 & -1.8487 & -1.8081 & -1.7529 & -1.6765 & -1.5684 & -1.4118 & - & - & - \\
\hline$E_{2.5}^{f d}$ & -2.4313 & -2.4159 & -2.3959 & -2.3693 & -2.3335 & -2.2845 & -2.2161 & -2.1184 & -1.9754 & -1.7601 & - & - \\
\hline$E_{3.0}^{f d}$ & -2.9395 & -2.9258 & -2.9080 & -2.8843 & -2.8523 & -2.8081 & -2.7461 & -2.6568 & -2.5250 & -2.3246 & -2.0095 & - \\
\hline$E_{3.5}^{f d}$ & -3.4455 & -3.4332 & -3.4172 & -3.3958 & -3.3667 & -3.3265 & -3.2696 & -3.1873 & -3.0649 & -2.8773 & -2.5796 & - \\
\hline$E_{4.0}^{f d}$ & -3.9502 & -3.9390 & -3.9244 & -3.9048 & -3.8782 & -3.8412 & -3.7886 & -3.7122 & -3.5978 & -3.4212 & -3.1388 & -2.6686 \\
\hline
\end{tabular}


TABLE VI: Ground state energies of fermion-dimer systems calculated by using $H_{2}$.

\begin{tabular}{|c|c|c|c|c|c|c|c|c|c|c|c|c|}
\hline$L$ & 17 & 16 & 15 & 14 & 13 & 12 & 11 & 10 & 9 & 8 & 7 & 6 \\
\hline$E_{1.5}^{f d}$ & -1.3967 & -1.3741 & -1.3449 & -1.3067 & -1.2561 & -1.1878 & -1.0941 & -0.9629 & - & - & - & - \\
\hline$E_{2.0}^{f d d}$ & -1.9148 & -1.8958 & -1.8710 & -1.8384 & -1.7947 & -1.7353 & -1.6528 & -1.5359 & -1.3663 & - & - & - \\
\hline$E_{2.5}^{f d}$ & -2.4267 & -2.4103 & -2.3887 & -2.3602 & -2.3217 & -2.2689 & -2.1951 & -2.0895 & -1.9346 & -1.7011 & - & - \\
\hline$E_{3.0}^{f d d}$ & -2.9353 & -2.9207 & -2.9015 & -2.8760 & -2.8414 & -2.7938 & -2.7266 & -2.6299 & -2.4868 & -2.2691 & -1.9264 & - \\
\hline$E_{3.5}^{f d d}$ & -3.4418 & -3.4286 & -3.4114 & -3.3883 & -3.3570 & -3.3136 & -3.2522 & -3.1631 & -3.0304 & -2.8269 & -2.5039 & - \\
\hline$E_{40}^{f d d}$ & -3.9470 & -3.9351 & -3.9193 & -3.8983 & -3.8696 & -3.8297 & -3.7730 & -3.6903 & -3.5665 & -3.3753 & -3.0694 & -2.5611 \\
\hline
\end{tabular}

TABLE VII: Ground state energy of dimers in the boosted frame calculated by using $H_{1}$.

\begin{tabular}{|c|c|c|c|c|c|c|c|c|c|c|c|c|}
\hline$L$ & 17 & 16 & 15 & 14 & 13 & 12 & 11 & 10 & 9 & 8 & 7 & 6 \\
\hline$E_{\vec{k}}^{1.5}$ & -1.5043 & -1.5066 & -1.5100 & -1.5150 & -1.5226 & -1.5338 & -1.5502 & -1.5741 & - & - & - & - \\
\hline$E_{\vec{k}}^{2 .} .0$ & -2.0020 & -2.0032 & -2.0051 & -2.0083 & -2.0134 & -2.0215 & -2.0343 & -2.0543 & -2.0855 & - & - & - \\
\hline$E_{\vec{k}}^{2 . .5}$ & -2.5009 & -2.5016 & -2.5028 & -2.5047 & -2.5081 & -2.5138 & -2.5235 & -2.5398 & -2.5666 & -2.6106 & - & - \\
\hline$E_{\vec{k}}^{3.0}$ & -3.0005 & -3.0009 & -3.0016 & -3.0028 & -3.0051 & -3.0091 & -3.0164 & -3.0293 & -3.0519 & -3.0911 & -3.1586 & - \\
\hline$E_{\vec{k}}^{3.5}$ & -3.5003 & -3.5005 & -3.5009 & -3.5017 & -3.5033 & -3.5062 & -3.5116 & -3.5218 & -3.5406 & -3.5750 & -3.6372 & - \\
\hline$E_{\vec{k}}^{4.0}$ & -4.0001 & -4.0003 & -4.0006 & -4.0011 & -4.0022 & -4.0042 & -4.0083 & -4.0164 & -4.0319 & -4.0619 & -4.1187 & -4.2258 \\
\hline
\end{tabular}

TABLE VIII: Ground state energy of dimers in the boosted frame calculated by using $H_{2}$.

\begin{tabular}{|c|c|c|c|c|c|c|c|c|c|c|c|c|}
\hline$L$ & 17 & 16 & 15 & 14 & 13 & 12 & 11 & 10 & 9 & 8 & 7 & 6 \\
\hline$E_{\vec{k}}^{1.5}$ & -1.5046 & -1.5070 & -1.5105 & -1.5158 & -1.5236 & -1.5351 & -1.5517 & -1.5758 & - & - & - & - \\
\hline$E_{\vec{k}}^{2.0}$ & -2.0022 & -2.0034 & -2.0055 & -2.0089 & -2.0142 & -2.0227 & -2.0359 & -2.0565 & -2.0881 & - & - & - \\
\hline$E_{\vec{k}}^{2.5}$ & -2.5010 & -2.5018 & -2.5030 & -2.5051 & -2.5087 & -2.5148 & -2.5250 & -2.5418 & -2.5694 & -2.6142 & - & - \\
\hline$E_{\vec{k}}^{3.0}$ & -3.0006 & -3.0010 & -3.0018 & -3.0031 & -3.0055 & -3.0099 & -3.0176 & -3.0312 & -3.0548 & -3.0952 & -3.1640 & - \\
\hline$E_{\vec{k}}^{3.5}$ & -3.5003 & -3.5005 & -3.5010 & -3.5019 & -3.5036 & -3.5067 & -3.5125 & -3.5234 & -3.5608 & -3.6225 & -3.7487 & - \\
\hline$E_{\vec{k}}^{4.0}$ & -4.0003 & -4.0005 & -4.0008 & -4.0014 & -4.0025 & -4.0048 & -4.0092 & -4.0178 & -4.0344 & -4.0660 & -4.1249 & -4.2350 \\
\hline
\end{tabular}

[1] G. V. Skornyakov and K. A. Ter-Martirosyan, Sov. Phys. JETP 4, 648 (1957).

[2] S. Bour, S. König, D. Lee, H. -W. Hammer and U. -G. Meißner, Phys. Rev. D84, 091503(R) (2011).

[3] V. Efimov, Phys. Lett. B33, 563 (1970).

[4] T. Kraemer et al., Nature 440, 315 (2006).

[5] C. Chin, R. Grimm, P. Julienne and E. Tiesinga, Rev. Mod. Phys. 82, 1225 (2010).

[6] E. Braaten and H. -W. Hammer, Phys. Rept. 428, 259 (2006).

[7] L. Platter, Few Body Syst. 46, 139 (2009).

[8] K. Helfrich, H. -W. Hammer, and D. S. Petrov, Phys. Rev. A 81, 042715 (2010).

[9] L. Thomas, Phys. Rev. 47, 903 (1935).

[10] D. S. Petrov, Phys. Rev. A 67, 010703 (2003).

[11] D. S. Petrov, C. Salomon, and G. V. Shlyapnikov, Phys. Rev. A 71, 012708 (2005).

[12] G. Rupak, arXiv:0605074 [nucl-th].

[13] J. von Stecher, C. H. Greene, and D. Blume, Phys. Rev. A 77, 043619 (2008).

[14] J. Levinsen, arXiv:0807.2840 [cond-mat.supr-con].

[15] J. Levinsen and D. S. Petrov, Eur. Phys. J. D 65, 67 (2011).

[16] S. Tan, Annals of Phys. 323, 2952 (2008). 
[17] W. Dilg, L. Koester, and W. Nistler, Phys. Lett. 36B, 208 (1971).

[18] P. F. Bedaque and U. van Kolck, Phys. Lett. B 428, 221 (1998).

[19] P. F. Bedaque, H. -W. Hammer, and U. van Kolck, Phys. Rev. C 58, R641 (1998).

[20] C. Lanczos, J. Res. Nat. Bur. Stand. 45, 255 (1950).

[21] D. Lee, B. Borasoy and T. Schäfer, Phys. Rev. C 70, 014007 (2004).

[22] D. Lee and T. Schäfer, Phys. Rev. C 73, 015202 (2006).

[23] D. Lee, Phys. Rev. Lett. 98, 182501 (2007).

[24] M. Lüscher, Commun. Math. Phys. 104, 177 (1986).

[25] M. Lüscher, Nucl. Phys. B 354, 531 (1991).

[26] S. R. Beane, P. F. Bedaque, A. Parreno, M. J. Savage, Phys. Lett. B 585, 106 (2004).

[27] Z. Davoudi and M. J. Savage, Phys. Rev. D 84, 114502 (2011).

[28] S. Kreuzer and H. -W. Hammer, Phys. Lett. B 673, 260 (2009).

[29] S. Kreuzer and H. -W. Hammer, Phys. Lett. B 694, 424 (2011).

[30] S. Kreuzer and H. Grießhammer, arXiv:1205.0277 [nucl-th].

[31] A. Rokash, E. Epelbaum, H. Krebs, D. Lee, work in progress.

[32] S. Beane, et al., Phys. Rev. D 85054511 (2012). 